\title{
Dynamical inference: Where phase synchronization and generalized synchronization meet
}

\author{
Tomislav Stankovski, Peter V. E. McClintock, and Aneta Stefanovska* \\ Department of Physics, Lancaster University, Lancaster, LA1 4YB, United Kingdom \\ (Received 11 July 2013; revised manuscript received 25 November 2013; published 10 June 2014)
}

\begin{abstract}
Synchronization is a widespread phenomenon that occurs among interacting oscillatory systems. It facilitates their temporal coordination and can lead to the emergence of spontaneous order. The detection of synchronization from the time series of such systems is of great importance for the understanding and prediction of their dynamics, and several methods for doing so have been introduced. However, the common case where the interacting systems have time-variable characteristic frequencies and coupling parameters, and may also be subject to continuous external perturbation and noise, still presents a major challenge. Here we apply recent developments in dynamical Bayesian inference to tackle these problems. In particular, we discuss how to detect phase slips and the existence of deterministic coupling from measured data, and we unify the concepts of phase synchronization and general synchronization. Starting from phase or state observables, we present methods for the detection of both phase and generalized synchronization. The consistency and equivalence of phase and generalized synchronization are further demonstrated, by the analysis of time series from analog electronic simulations of coupled nonautonomous van der Pol oscillators. We demonstrate that the detection methods work equally well on numerically simulated chaotic systems. In all the cases considered, we show that dynamical Bayesian inference can clearly identify noise-induced phase slips and distinguish coherence from intrinsic coupling-induced synchronization.
\end{abstract}

DOI: 10.1103/PhysRevE.89.062909

PACS number(s): 05.45.Xt, 02.50.Tt, 05.45.Tp

\section{INTRODUCTION}

Synchronization emerges from the interactions between oscillatory systems. It is a ubiquitous phenomenon that can involve either two or many systems, and it leads to the onset of coordinated dynamics and spontaneous order [1,2], with examples ranging from the synchronization of fireflies [3], through the cardiorespiratory system [4], to electrochemical oscillators [5]. Starting from Huygens, who first observed synchronization, many other studies and definitions have appeared, leading to different descriptions of synchronization [6]. Two of them, phase synchronization (PS) [1] and generalized synchronization (GS) [7], are of particular interest. They appeared during the era when dynamical chaos was also being intensively studied, and interest grew rapidly, especially given that synchronization can emerge from weak interactions. The markedly different definitions of PS [8] and GS [9] were published in the same volume of Physical Review Letters, with that of PS relying on the concept of phase locking, whereas GS related to the state amplitudes and the corresponding stability of the systems. Thus, PS and GS are applied to different domains of observables to describe what, as we will show below, is actually the same underlying phenomenon-synchronization.

It is often desirable to be able to detect synchronization from measured time series. This led to the development of a number of different methods for the detection of both PS and GS. The methods for PS detection require phase time series, and are based mostly on the statistics of the phase difference [10-13].

\footnotetext{
*aneta@lancaster.ac.uk
}

Published by the American Physical Society under the terms of the Creative Commons Attribution 3.0 License. Further distribution of this work must maintain attribution to the author(s) and the published article's title, journal citation, and DOI.
GS is assessed through use of the state signals of the interacting systems: different methods have been developed based on nearest-neighbor mapping [7], mutual information [14], generalized angle [15], and statistical modeling [16]. Parlitz et al. studied the connections and similarities between PS and GS [17]. All of these methods, however, detect the outcome from the statistics of the observables. Thus, they typically rely on detecting effects such as mutual information or transfer entropy, and many of them infer neither the noise intensity nor the parameters characterizing the noise-induced events. Dynamical inference $[18,19]$, on the other hand, can reveal the underlying dynamical mechanisms together with relevant parameter values, thus yielding deeper understanding of the synchronization process.

Two characteristic features of natural systems are their inherent time-variability and their mutual interactions. A new class of systems, named chronotaxic [20], has recently been introduced: nonautonomous self-sustained oscillators with time-varying, but stable, amplitudes and frequencies. Systems exhibiting such features are found in many different fields, including the cardiorespiratory system [4], climate [21], and evolutionary science [22], but they have often been treated as stochastic. Chronotaxic systems have been identified as highly deterministic and now need new analytic approaches, or modifications of the existing ones. The recently developed method for studying interoscillator interactions based on Bayesian dynamical inference $[18,19]$ is especially well suited to the treatment of systems with time-varying parameters, including, in particular, those that are chronotaxic. In what follows we extend this method to the detection of GS and we study the similarities between GS and PS and their mutual relationship.

Two synchronization detection methods, for PS and GS, respectively, are presented below and it is shown that they can improve the detection of "genuine" synchronization due to deterministic functional interactions. The proposed methods are based on a common inferential framework, which exploits dynamical Bayesian inference [18,23-25] of the time-evolving 
interactions in the presence of dynamical noise. PS is evaluated by testing whether the phase signals resemble a synchronized phase oscillator model, while GS is evaluated through the asymptotic stability of the inferred driven oscillator. We test and illustrate the equivalence and consistency of the two methods on signals emanating from an analog electronic simulation of two unidirectionally coupled nonautonomous van der Pol oscillators. The noise in the signals originates from the experimental environment and the imperfections of the electronic components. After demonstrating the application of the methods to analog signals, we apply them to numerical signals to point out two distinctive situations where they offer a significant advance over earlier approaches. We show that Bayesian dynamical inference is able to establish reliably whether observed phase slips are noise-induced or not. In the case of GS, the proposed Bayesian inference-based method can distinguish, consistently and precisely, whether two oscillators are simply coherent (but functionally independent), or intrinsically synchronized due to a mutual coupling. The Bayesian-based methods are also readily applicable to the case of chaotic dynamical systems. We illustrate the latter case by demonstrating satisfactory detection of PS and GS between coupled Rössler and Lorenz chaotic systems, with time-varying parameters and subject to noise.

Section II provides details of how the methods are developed. A brief description of dynamical Bayesian inference for time-evolving interactions is provided, followed by an explanation of how PS and GS are evaluated from the parameters that are inferred. In Sec. III the analog experiment is described and the methods to detect both PS and GS are brought to bear on the same signal originating from it. Section IV outlines how both methods can detect whether the existence of phase slips is just on account of the perturbing effect of noise or is on account of interactions. In Sec. V we demonstrate that GS method can distinguish between coherence and intrinsic coupling-induced synchronization. Section VI provides a comprehensive discussion of applications to coupled chaotic systems. Finally, we summarize and draw conclusions in Sec. VII. The Appendix describes succinctly how the measurement noise can be treated within the same inferential framework.

\section{DYNAMICAL INFERENCE OF COUPLED OSCILLATORS}

\section{A. Time-evolving dynamical Bayesian inference}

In the present context, dynamical inference refers to a procedure for creating a model in terms of differential equations from an analysis of time-series. This is quite different [26] from other statistical methods, which measure quantities such as entropy, Granger causality, and conditional probability [27-31]. The most important distinction is that the end results of dynamical inference are the equations of motion. These (ordinary or stochastic) differential equations can describe in full not only the effects observed but also the underlying dynamical mechanisms, phenomena, and qualitative transitions. In this way, dynamical inference methods can probe the effective connectivity [32] between the coupled oscillators.

The dynamical inference technique applied here uses a recently introduced method based on a Bayesian frame- work $[18,19]$. The main feature is that the method is applied to a stochastic differential model whose deterministic part is allowed to be time-varying. The aim is to provide a way of detecting synchronization in a model of two (or more) weakly interacting oscillators subject to noise. Such a model is described by the stochastic differential equation

$$
\dot{\chi}_{i}=\mathbf{f}\left(\chi_{i}, \chi_{j} \mid \mathbf{c}\right)+\sqrt{\mathbf{D}} \xi_{i},
$$

where $i \neq j=1,2$, and $f\left(\chi_{i}, \chi_{j} \mid \mathbf{c}\right)$ are base functions representing the deterministic part of the internal and the interacting dynamics. The vector $\mathbf{c}$ denotes the parameters acting as scale coefficients for the base functions. The dynamical noise is assumed to be white, Gaussian, and parametrized by a noise diffusion matrix D. We focus on inferring the dynamical noise because of its ability to induce phase-slips that can affect synchronization detection directly. Note, however, that it is also possible to infer the measurement noise within the same framework: see the Appendix and Ref. [33]. At this point we speak of $\chi_{i}$ in general, but later we will refer separately to the phase or state domain depending on the type of synchronization we are treating, i.e., phase or generalized, respectively.

Given that $2 \times M$ time-series $\mathcal{X}=\left\{\chi_{n} \equiv \chi\left(t_{n}\right)\right\}\left(t_{n}=n h\right)$ are provided, and assuming that the model base functions are known, the main task for dynamical Bayesian inference $[24,33]$ is to infer the unknown model parameters and the noise diffusion matrix $\mathcal{M}=\{\mathbf{c}, \mathbf{D}\}$. The problem eventually reduces to maximization of the conditional probability to observe the parameters $\mathcal{M}$, given the data $\mathcal{X}$. For this we applied Bayes' theorem, which exploits the prior density $p_{\text {prior }}(\mathcal{M})$ of the parameters and the likelihood function $\ell(\mathcal{X} \mid \mathcal{M})$ to observe $\mathcal{X}$ given the choice $\mathcal{M}$, in order to determine the posterior density $p_{\mathcal{X}}(\mathcal{M} \mid \mathcal{X})$ of the unknown parameters $\mathcal{M}$ conditioned on the observations $\mathcal{X}$ :

$$
p_{\mathcal{X}}(\mathcal{M} \mid \mathcal{X})=\frac{\ell(\mathcal{X} \mid \mathcal{M}) p_{\text {prior }}(\mathcal{M})}{\int \ell(\mathcal{X} \mid \mathcal{M}) p_{\text {prior }}(\mathcal{M}) d \mathcal{M}} .
$$

The prior distribution, enclosing previous knowledge of the unknown parameters based on observations, is assumed to be known. The task is therefore to determine the likelihood functions in order to infer the final posterior result. If the sampling $h$ is small enough, ${ }^{1}$ then one can construct the Euler midpoint approximation of Eq. (1) for the acquired time-series:

$$
\chi_{i, n+1}=\chi_{i, n}+h f\left(\chi_{i, n}^{*}, \chi_{j, n}^{*} \mid \mathbf{c}\right)+h \sqrt{\mathbf{D}} \mathbf{z}_{n} .
$$

Here $\chi_{n}^{*}=\left(\chi_{n+1}+\chi_{n}\right) / 2$ and $\mathbf{z}_{n}$ is the stochastic integral of the noise term over time: $\mathbf{z}_{n} \equiv \int_{t_{n}}^{t_{n+1}} \mathbf{z}(t) d t=\sqrt{h} \mathbf{H} \xi_{n}$ for the $\mathbf{H}$ matrix that satisfies the Cholesky decomposition $\mathbf{H H}^{\mathbf{T}}=$ D. Use of the stochastic integral for noise that is white and independent leads to the likelihood function, which is given by a product over $n$ of the probability of observing $\chi_{n+1}$ at each time ([33] and references within). The negative log-likelihood

\footnotetext{
${ }^{1}$ With a large mismatch between the integration and sampling rates, the inference of the stochastic part could become less precise. Given the high sampling rates provided by current (and foreseeable) analog-to-digital converters, however, this approximation is already appropriate in most experimental applications.
} 
function is then $S=-\ln \ell(\mathcal{X} \mid \mathcal{M})$, given as

$$
\begin{aligned}
S= & \frac{N}{2} \ln |\mathbf{D}|+\frac{h}{2} \sum_{n=0}^{N-1}\left\{\mathbf{c}_{k} \frac{\partial \mathbf{f}_{k}\left(\chi_{\cdot, n}\right)}{\partial \chi}\right. \\
& \left.+\left[\dot{\chi}_{n}-\mathbf{c}_{k} \mathbf{f}_{k}\left(\chi_{\cdot, n}^{*}\right)\right]^{T}\left(\mathbf{D}^{-1}\right)\left[\dot{\chi}_{n}-\mathbf{c}_{k} \mathbf{f}_{k}\left(\chi_{\cdot, n}^{*}\right)\right]\right\},
\end{aligned}
$$

with implicit summation over the repeated index $k$ and approximated derivatives $\dot{\chi}_{n}=\left(\chi_{n+1}-\chi_{n}\right) / h$. The log-likelihood function Eq. (3) is of quadratic form. Thus, if the prior is a multivariate normal distribution, so also will be the posterior. Given such a distribution as a prior for the parameters c, with mean $\overline{\mathbf{c}}$, and covariance matrix $\Sigma_{\text {prior }} \equiv \Xi^{-1}$ prior , the final stationary point of $S$ is calculated recursively from

$$
\begin{aligned}
\mathbf{D} & =\frac{h}{N}\left[\dot{\chi}_{n}-\mathbf{c}_{k} \mathbf{f}_{k}\left(\chi_{\cdot, n}^{*}\right)\right]^{T}\left[\dot{\chi}_{n}-\mathbf{c}_{k} \mathbf{f}_{k}\left(\chi_{\cdot, n}^{*}\right)\right], \\
\mathbf{c}_{k} & =\left(\Xi^{-1}\right)_{k w} \mathbf{r}_{w}, \\
\mathbf{r}_{w} & =\left(\Xi_{\text {prior }}\right)_{k w} \mathbf{c}_{w}+h \mathbf{f}_{k}\left(\chi_{\cdot, n}^{*}\right)\left(\mathbf{D}^{-1}\right) \dot{\chi}_{n}-\frac{h}{2} \frac{\partial \mathbf{f}_{k}\left(\chi_{\cdot, n}\right)}{\partial \chi}, \\
\Xi_{k w} & =\left(\Xi_{\text {prior }}\right)_{k w}+h \mathbf{f}_{k}\left(\chi_{\cdot, n}^{*}\right)\left(\mathbf{D}^{-1}\right) \mathbf{f}_{w}\left(\chi_{\cdot, n}^{*}\right),
\end{aligned}
$$

where summation over $n=1, \ldots, N$ is assumed and the summation over repeated indices $k$ and $w$ is again implicit. The initial prior can be set as the noninformative flat normal distribution, $\Xi_{\text {prior }}=0$ and $\overline{\mathbf{c}}_{\text {prior }}=0$. These four Eqs. (4) are applied to a single block of data $\mathcal{X}$ and the posterior multivariate probability $\mathcal{N}_{\mathcal{X}}(c \mid \bar{c}, \Xi)$ explicitly defines the probability density of each parameter set of the model Eq. (1).

In dynamical Bayesian inference each new prior distribution depends on and uses the previously inferred posterior distribution. In this way, our inference uses informative priors and differs from most of the maximum likelihood estimators. In our framework, however, this information propagation is amended in order to follow the time-variability of the parameters [18]. The new prior covariance matrix becomes $\Sigma_{\text {prior }}^{n+1}=\Sigma_{\text {post }}^{n}+\Sigma_{\text {diff }}^{n}$, where $\Sigma_{\text {diff }}^{n}$ describes which part of the dynamical fields defining the oscillators has changed, and the size of the change. Constructed in this way, the prior will lie within the clearly defined boundaries [19] between the case of full information propagation $\Sigma_{\text {prior }}^{n+1}=\Sigma_{\text {post }}^{n}$ and that of no propagation $\Sigma_{\text {prior }}^{n+1}=\infty$.

The description above, and in the rest of the paper, is for two interacting oscillators. Nonetheless, the theory also holds for a larger number of oscillators-see, e.g., the generalization to networks in [19]. Dynamical Bayesian inference can be applied successfully to a relatively wide spectrum of physical problems but it is not, of course, applicable to every experimental problem. In certain cases (e.g., when very fast processing is required, or the measurement noise is very high, or the data blocks are extremely small), it is possible that an alternative form of dynamical inference [34-37] may cope better with some of the inferential issues and can then be used for detection of PS and GS in the novel way presented below. A tutorial about the practical implementation of dynamical Bayesian inference, including programming and software codes from which the following examples can be easily reproduced, is provided in Ref. [38].

\section{B. Detection of phase synchronization}

To detect PS we perform inference in the phase domain; i.e., we use phase time-series derived from the interacting oscillators. Depending on the nature of the state signals, the instantaneous phases can be estimated by use of any one of a number of different phase detection methods [34,39-41]. If the oscillators are weakly interacting, their dynamics can be approximated by a phase oscillator model [42]. This serves as the base model for the Bayesian inference:

$$
\dot{\phi}_{i}=\mathbf{f}\left(\phi_{i}, \phi_{j} \mid \mathbf{c}\right)+\sqrt{\mathbf{D}} \xi_{i},
$$

where $\phi_{i}$ are the phase variables. Because of their oscillatory nature and the existence of periodic solutions, the base functions $\mathbf{f}$ can effectively be decomposed into Fourier series [18]:

$$
\dot{\phi}_{i}=\sum_{k=-K}^{K} c_{k}^{(i)} \Phi_{i, k}\left(\phi_{1}, \phi_{2}\right)+\xi_{i}
$$

where $i=1,2, \Phi_{1,0}=\Phi_{2,0}=1, c_{0}^{(i)}=\omega_{i}$, and the other $\Phi_{i, k}$ and $c_{k}^{(i)}$ are the $K$ most important Fourier components. Note that in this way we do not prescribe any specific model, and the inference is applicable to interacting oscillators quite generally.

Once we have inferred the parameters $\mathcal{M}=\{\mathbf{c}, \mathbf{D}\}$, our goal is to check whether or not the deterministic phase oscillator model Eq. (5) undergoes PS for those parameters c. We therefore present the phase dynamics on a $\mathbb{T}^{2}$ torus defined by the toroidal coordinate $\zeta(t)=\phi_{1}(t)+\phi_{2}(t)$ and polar coordinate $\psi(t)=\phi_{1}(t)-\phi_{2}(t)$. Next, we consider a Poincaré section defined by $\zeta=0$ and assume that $d \zeta(t) /\left.d t\right|_{\zeta=0}>0$ for all $\psi$. In this way we construct a map $M:[0,2 \pi] \rightarrow$ $[0,2 \pi]$ that defines, for each $\psi_{n}$ on the Poincaré section, the next phase $\psi_{n+1}$ after one period of the toroidal coordinate $\psi_{n+1}=M\left(\psi_{n}\right)$. Synchronization is verified if $\psi_{e}$ exists such that $\psi_{e}=M\left(\psi_{e}\right)$ and $\left|d M\left(\psi_{e}\right) / d \psi\right|<1$. The existence of a fixed solution is established by application of a modified Newton's root-finding method. A detailed description of the procedure is given in Ref. [19]. If there is a root, the oscillators are synchronized $I_{\mathrm{PS}}=1$; otherwise they are not and $I_{\mathrm{PS}}=0$.

\section{Detection of generalized synchronization}

To detect GS we perform inference in the state domain using the "raw" signals for the whole state space. In doing so, we assess the amplitude dynamics of the interacting oscillators. The model to be inferred is then expressed as

$$
\dot{\mathbf{x}}_{i}=\mathbf{f}\left(\mathbf{x}_{i}, \mathbf{x}_{j} \mid \mathbf{c}\right)+\sqrt{\mathbf{D}} \xi_{i},
$$

with $\mathbf{x}_{i}$ being the state variables. We will assume that the deterministic model and the base functions $\mathbf{f}$ are known and presented as polynomial functions. In general, it is nontrivial to determine the state model underlying the dynamics of a given time series. Nonetheless, techniques do exist for treating this problem-for example, the variational Bayesian or the polynomial best fit methods [24,36,43]. These are model selection methods that start from a large set of base functions and try to establish the most probable approximative model representing the dynamics of the observed time-series. On the other hand, there also exist cases where the model is known 
a priori and the main task of the dynamical inference is then to reveal the temporal dynamics from the time series, e.g., a recent application of the same inference framework to effect secure communications [44].

Next, we use the inferred parameters $\mathcal{M}=\{\mathbf{c}, \mathbf{D}\}$ and the state model to detect whether or not the two systems are synchronized. To do so, we employed a theorem by Kocarev and Parlitz [9] stating that a unidirectionally coupled system is in a state of GS if the driven system is asymptotically stable. Knowing the model and the parameters, one can evaluate the largest Lyapunov exponent (LLE) of the driven oscillator $[45,46]$. If the LLE is negative, then the driven oscillator is asymptotically stable and the two oscillators undergo GS. Hence, the LLE can serve as a synchronization index. Moreover, due to the information propagation within the inference method, one is able to follow the time variability of the LLE, and hence the time-evolution and corresponding transitions into or out of GS.

\section{DETECTING PHASE AND GENERALIZED SYNCHRONIZATION THROUGH A COMMON INFERENTIAL FRAMEWORK}

\section{A. Analog simulation}

As Bayesian dynamical inference is by definition stochastic, one often encounters the question: Is the inference valid under noiseless conditions? In theory, in the complete absence of noise, the zero-noise matrix $\mathbf{D}=0$ in Eq. (4) will result in a singularity. Consequently, if one applies inference to numerically generated signals with virtually no noise, the method will fail in execution. We argue, however, that such a situation is (almost) never encountered under real experimental conditions. There will inevitably always be at least a little noise (the omnipresent thermal noise if nothing else), which will allow the methods to function correctly. To illustrate this we performed an analog study of two interacting systems, without using an explicit noise generator.
Analog experiments have been used extensively for studying the dynamics of nonlinear systems [47-52]. They provide a convenient way to study the continuous dynamics and interactions between oscillatory systems and stochastic processes in real time. The uncertainty in the system, arising from the noise embedded in the signals, has a more realistic meaning here, usually being attributed to environmental disturbances or to imperfections of some of the electronic components in the circuits. This is an example of the dynamical noise that is the main focus of this manuscript. In addition, during the process of data acquisition and discretization, measurement noise is introduced that has no links with the actual dynamics of the oscillators. In respect of synchronization, dynamical noise can introduce phase slips, whereas measurement noise will only reduce the overall precision of the inference.

The model system to be investigated consists of two coupled van der Pol oscillators:

$$
\begin{aligned}
& \frac{1}{c^{2}} \ddot{x}_{1}-\mu_{1}\left(1-x_{1}^{2}\right) \frac{1}{c} \dot{x}_{1}+\left[\omega_{1}+s(t)\right]^{2} x_{1}=0 \\
& \frac{1}{c^{2}} \ddot{x}_{2}-\mu_{2}\left(1-x_{2}^{2}\right) \frac{1}{c} \dot{x}_{2}+\omega_{2}^{2} x_{2}+\varepsilon x_{1}^{3}=0,
\end{aligned}
$$

where $i=1,2$ and $x_{i}$ are the state variables describing the dynamics of each subsystem, $\mu_{i}$ are the shape parameters that define the relaxation of each of the oscillator, and $\varepsilon$ is the coupling amplitude. Note that there is no explicit noise generator in Eq. (8). When the shape parameters are small $(\mu \rightarrow 0), \omega_{i}$ are the oscillator frequencies. The constant $c$ appears from each integration procedure and is introduced for electrical stability. The first oscillator has a nonautonomous term $s(t)$, which makes its frequency time-varying. The two oscillators are unidirectionally coupled such that the first oscillator drives the second one.

Figure 1 provides a block-diagram of the analog electronic implementation of the system under investigation [Eq. (8)]. All the operational amplifiers are type MC1458N, while the four-quadrant analog multipliers AD534LD. The output of

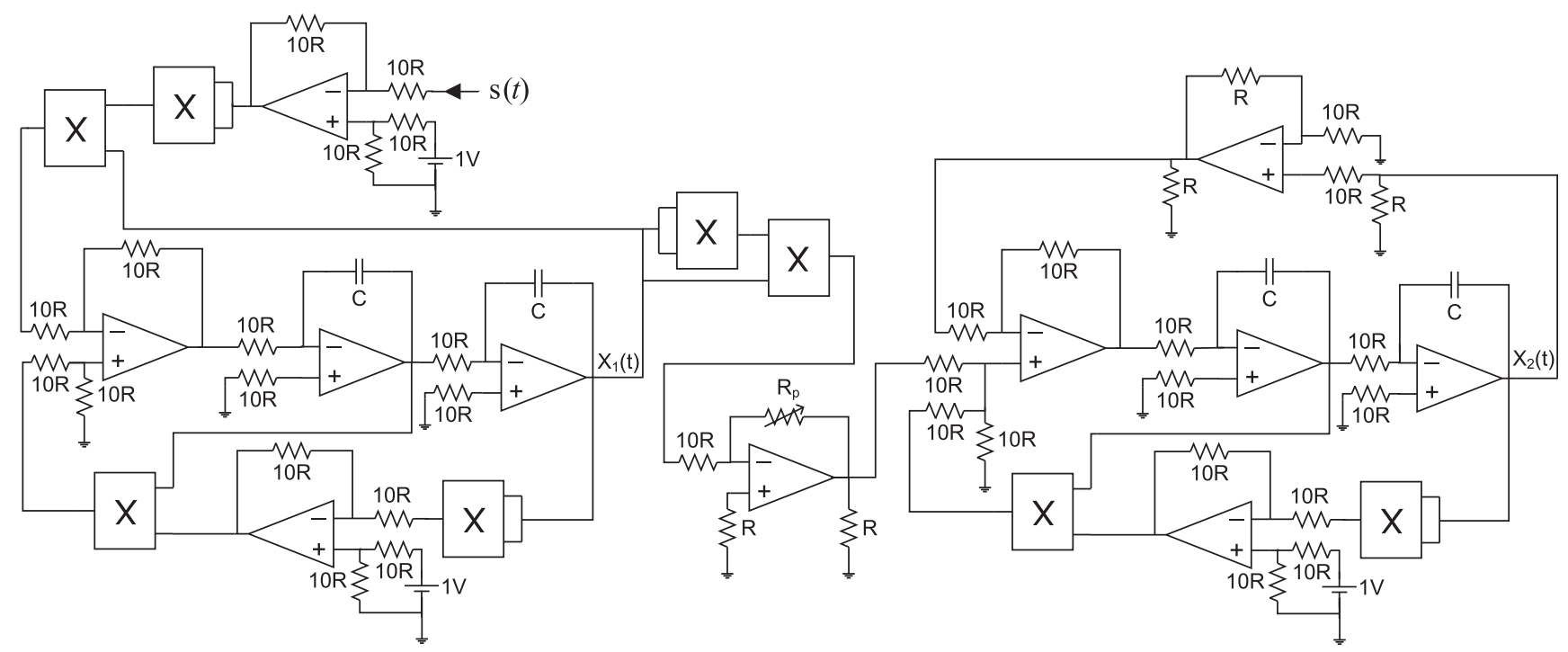

FIG. 1. Schematic block diagram of the analog electronic circuit used to model a pair of unidirectionally coupled, nonautonomous, van der Pol oscillators. Standard notation is used: the triangles correspond to amplifiers and the rectangles to multipliers. The resistance $R=1 \mathrm{k} \Omega$, the capacitance is $C=1 \mu \mathrm{F}$, and the resistance of the potentiometer $R_{p}=1 \rightarrow 10 \mathrm{k} \Omega$. 
each multiplier is automatically divided by a factor of 10 , and this is taken into account in the design of the circuit. For the design in Fig. 1 one can readily determine the values of the corresponding parameters in the system Eq. (8). The shape parameters are both set to unity $\mu_{1}=\mu_{2}=1$ and the basic frequencies are $\omega_{1}=1$ and $\omega_{2}=1.1$. A nonautonomous influence is introduced additively in the frequency of the first oscillator by means of a periodic sine or square-wave signal from an analog signal generator. The constant $c=100$. Thus, the true oscillating frequencies are $f_{1}=\omega_{1} 100 / 2 \pi=$ $15.92 \mathrm{~Hz}$ and $f_{2}=\omega_{2} 100 / 2 \pi=17.51 \mathrm{~Hz}$. The analog-todigital conversion is performed at a sampling frequency $f_{s}=$ $1000 \mathrm{~Hz}$. By varying the resistance $R_{p}$ of the potentiometer one can change the coupling strength $\varepsilon=0 \rightarrow 1$ - corresponding to a change from zero coupling to moderate coupling between the two interacting oscillators. In this way, one is able to observe the time-evolution of the dynamics and follow the synchronization transitions in real time.

Figure 2 shows examples of signals from the analog simulator. Because of the sinusoidal periodic influence on the frequency, the phase portrait (a) and the Lissajous curve (b) are not closed curves but, rather, oscillate within a confined region. The wavelet transforms [53,54] in Figs. 2(d) and 2(e) of the digitized signal $x_{1}$ in Fig. 2(c), show clearly the sinusoidal and square-wave time-variations of the frequency-a property often observed in (thermodynamically open) biological systems $[4,18]$. The square-wave forcing produces time variations
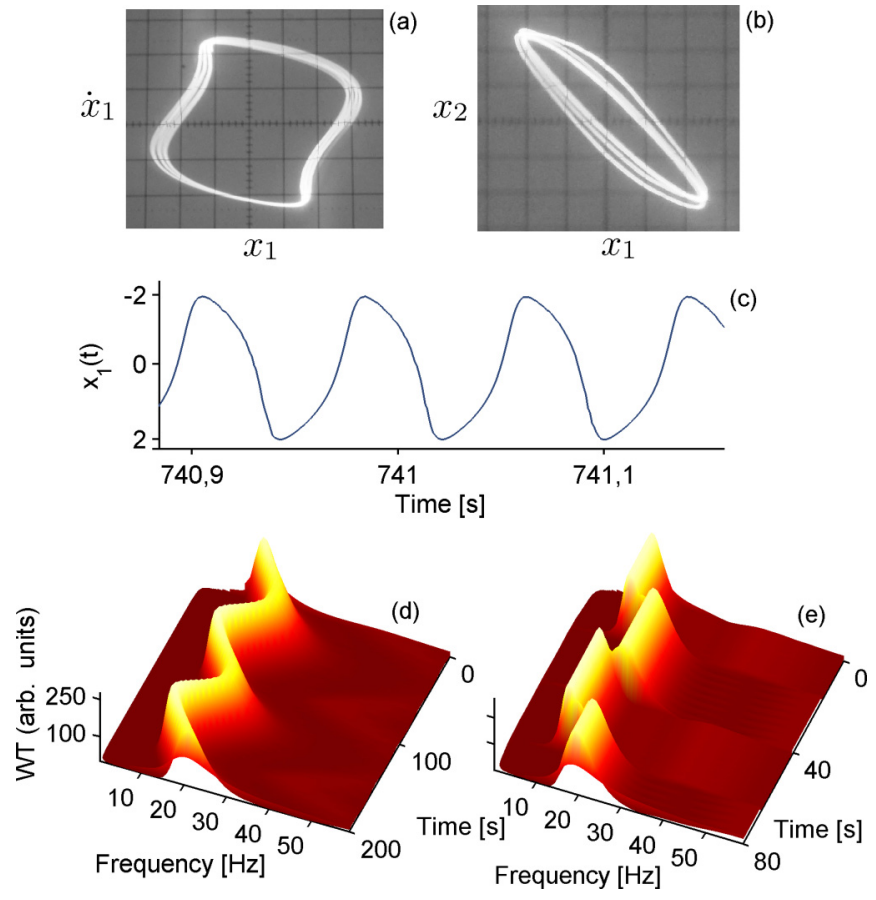

FIG. 2. (Color online) Some results from the analog electronic simulation of model Eq. (8). (a) Typical signals (oscilloscope traces) showing for a window in time the phase portrait of the first oscillator $x_{1}$ for a sinusoidal external force $s(t)$. (b) Oscilloscope trace of the Lissajous curve during synchronization. (c) The $x_{1}$ signals after A/D conversion. (d) Time-frequency wavelet transform of $x_{1}$ for a sinusoidal external force. (e) Wavelet transform of $x_{1}$ for a square-wave external force. resulting in synchronization transitions that can be observed in real time [55].

\section{B. Equivalence of phase and generalized synchronization}

The relationship between PS and GS was first analyzed by Parlitz et al. [17]. They outlined the main similarities and relations by detecting PS through phase difference and mean frequency and by detecting GS through the nearestneighbors test. Here, we detect PS and GS through the common inferential framework, where the evaluation of the synchronization state relies on dynamical models and the respective definitions of synchronization.

We applied the methods described above to analog signals from the unidirectionally coupled model Eq. (8). The frequency of the first oscillator was varied by the square-wave signal of Fig. 3(a). The variations were such that, for its low frequency state, the two oscillators were synchronized. Due to the periodicity of the external signal $s(t)$, however, intermittent synchronization occurred. First we analyzed PS. The phases were estimated by application of the Hilbert [39] and protophase-phase [34] transformations to the $x_{1}(t)$ and $x_{2}(t)$ signals. By decomposing the phase dynamics to second order in Fourier series, we inferred the parameters $\mathbf{c}$ of the interacting systems. Next, by reconstruction of the map using torus phase notation, we determined whether or not the coupled phase oscillators were synchronized, for each inferred set of parameters. The results in Fig. 3(b) show that we detected PS time variations and transitions consistently with the frequency variations. We then sought and detected GS. As base functions we used the polynomials on the left-hand side of Eq. (8). We
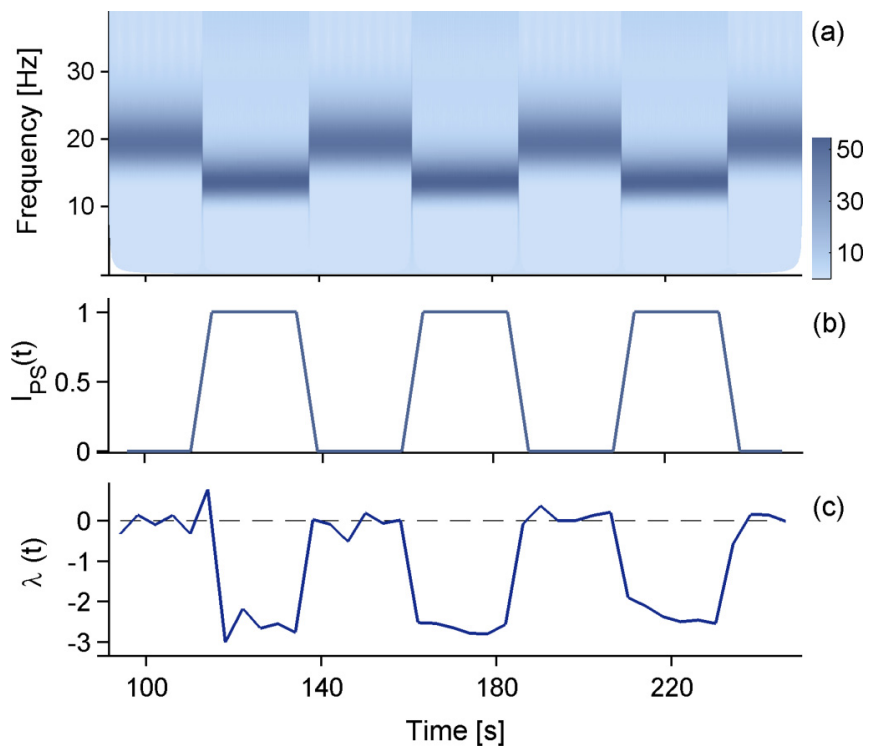

FIG. 3. (Color online) Detection of PS and GS from the same signals generated by analog simulation of the model Eq. (8). (a) Timefrequency wavelet transform of $x_{1}$. Note the discrete time-variation of the frequency due to the square-wave signal perturbation $s(t)$. (b) The detected PS index $I_{\mathrm{Ps}}$. High values of $I_{\mathrm{PS}}=1$ indicate synchronized intervals. (c) Largest Lyapunov exponent of the $x_{2}$ oscillator. Negative (nonzero) $\lambda(t)$ indicates asymptotic stability of the $x_{2}$ system and the occurrence of GS. Both cases were calculated with windows of $4 \mathrm{~s}$. 
applied the inference method to the four state signals created from the parameter set $\mathbf{c}$ for each block of data. Evaluation of the LLE $\lambda(t)$ then revealed whether the driven oscillator $x_{2}$ was asymptotically stable, and hence whether GS existed or not. The results in Fig. 3(c) show the time intervals of GS when the exponent was negative, and the nonsynchronized intervals where $\lambda$ was around zero and the driven oscillator is marginally stable. Again the detected GS transitions are consistent with the frequency variations.

It is evident that the detected PS and GS in Fig. 3 [cf. Figs. 3(b) and 3(c)] are mutually self-consistent and that they undergo essentially the same time evolution and regular transitions. Thus, our dynamical Bayesian inference, applied to different observables in the same system, and exploiting different synchronization definitions, has detected the same phenomenon in each case-synchronization.

\section{DETECTING NOISE-INDUCED PHASE SLIPS}

Noise can be the instigator of many events and phenomena in dynamical systems. It is especially important in relation to systems that are in "borderline" states [18,56,57]. Due to the importance of the latter, there is an increasing need for methods that can detect and describe the nature of such events.

We now try to describe the nature of phase slips in relation to PS and GS. In doing so, we use a numerical simulation of the same model Eq. (8), but with an explicit white-noise source and without the integration constant $c$ :

$$
\begin{aligned}
& \ddot{x}_{1}-\mu_{1}\left(1-x_{1}^{2}\right) \dot{x}_{1}+\left[\omega_{1}+s(t)\right]^{2} x_{1}+\xi_{1}(t)=0, \\
& \ddot{x}_{2}-\mu_{2}\left(1-x_{2}^{2}\right) \dot{x}_{2}+\omega_{2}^{2} x_{2}+\varepsilon x_{1}^{3}+\xi_{2}(t)=0 .
\end{aligned}
$$

The frequency parameters were $\omega_{1}=1.8, \omega_{2}=2.2, \mu_{1}=$ $\mu_{2}=1, \varepsilon=0.5$, and the noise strength was $D_{1}=D_{2}=$ 0.075 . Again the frequency of the first oscillator is perturbed by a square-wave signal $s(t)$, causing a regular sequence of synchronization intervals each of $500 \mathrm{~s}$. Moderate noise causes phase-slips during the synchronized intervals where the deterministic parameters (in particular the frequencies and couplings) are such that the oscillators are weakly synchronized, i.e., inside the Arnold tongue, but close to its border. This situation is illustrated by the simple phase difference in Fig. 4(a). There are two $500 \mathrm{~s}$ plateau-like intervals indicating synchrony. However, the two enlarged insets show that, during these intervals, phase-slips occur. Our objective is to ascertain whether these phase slips are noise-induced or interaction-induced.

To illustrate their effectiveness and advantages, we compare our methods with two earlier approaches based on the statistics of the observables. First we study PS. As part of the class of PS methods based on statistics of the phase difference [10-13], we use the synchronization index, based on the conditional probability [10], which we denote as $I_{\mathrm{CP}}$. The method first divides each phase interval into $N$ bins. Then, for each bin $l$, we calculate the dependence of $\phi_{2}\left(t_{j}\right)$, such that $\phi_{1}\left(t_{j}\right)$ belongs to this bin $l$, and $M_{l}$ is the number of points in the bin. The average over all bins leads to the final index:

$$
I_{\mathrm{CP}}\left(t_{j}\right)=1 / N \sum_{l=1}^{N}\left|M_{l}^{-1} \sum e^{i \phi_{2}\left(t_{j}\right)}\right| .
$$

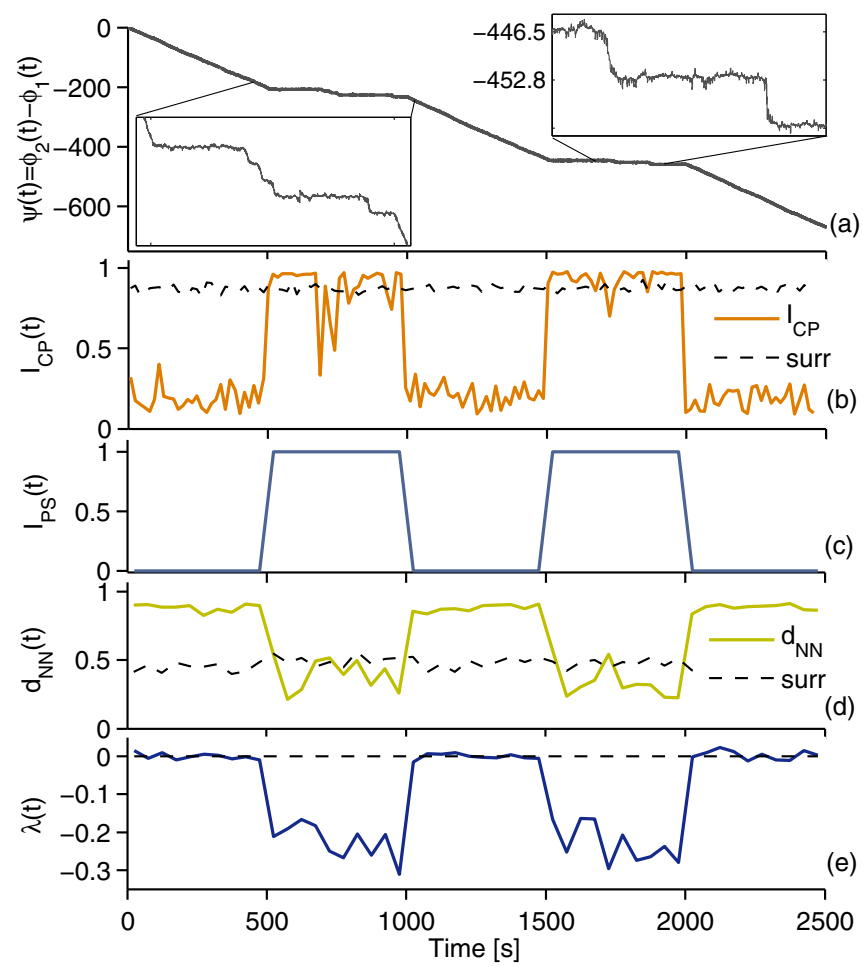

FIG. 4. (Color online) Detection of PS and GS for the model Eq. (9) in the presence of noise-induced phase slips. (a) The instantaneous phase difference. The insets are enlargements of the regions where the noise-induced phase slips occur. (b) The synchronization index based on the conditional probability $I_{\mathrm{CP}}$. The dashed line is the acceptance threshold for synchronization and denotes the mean +2 SD (standard deviations) of 100 phase cyclic surrogate [58] realizations. (c) The synchronization index for PS based on dynamical inference $I_{\mathrm{PS}}$. (d) Nearest-neighbor distance as a measure of GS. The dashed line is the acceptance threshold and denotes the mean -2 SD of 100 AAFT surrogate [59] realizations. (e) The dynamically inferred LLE $\lambda$ as a measure of GS. We note that $\lambda$ and $I_{\mathrm{PS}}$ have natural acceptance thresholds so that surrogate testing is not needed.

In this way, $I_{\mathrm{CP}}$ measures the conditional probability for $\phi_{2}$ to have a certain value provided $\phi_{1}$ is in a particular bin. We took $N=10$ as the number of bins, with windows of length $20 \mathrm{~s}$ and we used surrogate testing to determine statistical significance. The results shown in Fig. 4(b) demonstrate that the index $I_{\mathrm{CP}}$ was below the surrogate threshold for the time windows where the phase slips occurred. This would seem to suggest that the oscillators were unsynchronized throughout the whole of each 500 -s synchronization interval. However, computation of $I_{\mathrm{PS}}$ by our dynamical inference method, Fig. 4(c), shows that, actually, the oscillators remain synchronized throughout the whole of each 500-s interval. It is because of the noise being decomposed separately that our evaluation of the intrinsic parameters can reveal this clear conclusion [18] that the observed phase slips are noise-induced.

Let us now investigate the detection of GS in relation to such phase slips. We use the index based on nearest neighbors [17] — which starts from a delay-embedded state and measures if the neighboring states of the drive $\mathbf{u}^{\mathbf{n}}$ are 
mapped to neighbors in the response system $\mathbf{v}^{\mathbf{n}}$. The distance,

$$
d_{N N}=\frac{1}{N \delta} \sum_{n=1}^{N}\left\|\mathbf{v}^{\mathbf{n}}-\mathbf{v}^{\mathbf{n n}}\right\|,
$$

is normalized to show zero for synchronized states and one otherwise. We used three embedding dimensions, a delay of $\tau=20 \mathrm{~s}$, and windows of $50 \mathrm{~s}$. Figure 4(d) shows again that, due to the phase slips, the distance rises above the surrogate threshold indicating nonsynchronization. Note also that due to the strong noise the distance index does not attain either zero or one. The LLEs evaluated for the dynamically inferred parameters, on the other hand, are negative throughout the whole of each synchronization interval, indicating that the systems are intrinsically synchronized.

\section{DETECTING INTRINSIC SYNCHRONIZATION}

Synchronization is by definition an "adjustment of rhythms due to weak coupling" [1]. For synchronization to occur, therefore, there needs to be coupling, i.e., synchronization should be a consequence of interactions between the systems. This also implies that a coincidence of rhythms is not in itself sufficient to prove the existence of synchronization.

It is important to note that such situations can be encountered in real systems. For example if one studies the apparent cardiorespiratory synchronization between the respiration of one human subject with the cardiac activity of a different subject (as is done in creating intersubject surrogates), what appear to be short synchronized intervals can be detected using the available methods. Such results are clearly fallacious, given that the two signals cannot be related in any way at all — coming, as they do, from different subjects measured at different times.

To study this issue, we again used the numerical model Eq. (9), but with the two van der Pol oscillators set to the same frequency $\omega_{1}=\omega_{2}=0.9$. At intervals of $500 \mathrm{~s}$ we then switched the coupling $\varepsilon\{1\}=0.35$ off or on to produce sequential changes between the coherent and intrinsically synchronized states. The noise strength was $D_{1}=D_{2}=0.01$. Figure 5(a) shows the resultant phase difference. As expected, it is mostly constant, except for the noise perturbations and the small transients at the switching points between the intervals. These transients result from the nonlinear nature of the oscillators and act as initial states for the nonsynchronized intervals. Nevertheless, the phase difference is not diverging and it remains confined within $\pm 2 \pi$. The evaluation of the synchronization index $I_{\mathrm{CP}}$ is based directly on the statistics of the phase difference; consequently, it will not be able to distinguish between the two states. This is illustrated in Fig. 5(b) - the index is above the surrogate threshold throughout the whole time span, without a trace of distinction between the two states. Similarly, the nearest-neighbor test for GS is unable to detect the difference between the coherent and synchronized states. This is because the distance in the coherent state is again minimal and nondiverging. Figure 5(c) shows that the distance index is always below the surrogate threshold-indicating a constantly synchronized state. The only exceptions are the few points resulting from the switching between intervals. On the other hand, when we applied our

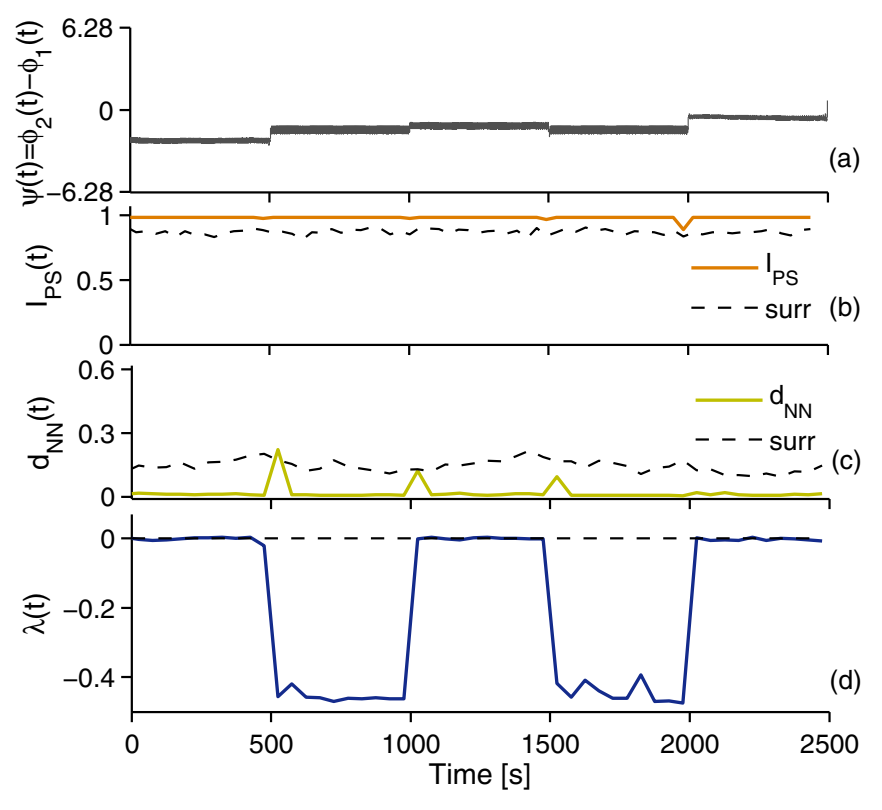

FIG. 5. (Color online) Distinction between coherent and intrinsically synchronized states in the model Eq. (9). (a) Instantaneous phase difference. (b) Synchronization index based on conditional probability $I_{\mathrm{CP}}$. The dashed line denotes mean $+2 \mathrm{SD}$ of 100 surrogate realizations. (c) Nearest-neighbor distance as a measure of GS. The dashed line is acceptance threshold and denotes mean 2 SD of 100 surrogate realizations. (d) Dynamically inferred LLE $\lambda$ as a measure of GS.

method for GS detection we were able clearly and precisely to distinguish the synchronized from the nonsynchronized (coherent only) states [Fig. 5(d)].

Because the phase domain in the coherent state does not fill enough space for inference, the $I_{\mathrm{PS}}$ index is not very reliable in distinguishing this as a nonsynchronized state. For cases with higher noise, when more of the space is spanned, the $I_{\mathrm{PS}}$ index will work consistently, but not in general.

\section{THE EASE OF INFERRING CHAOTIC INTERACTIONS}

In the preceding sections we focused on the synchronization of regular limit-cycle oscillators whose dynamics is time varying. The reason and motivation for doing so resulted from the great importance of such time-varying and chronotaxic systems [20] and their abundance in nature and biology. In order to illustrate the comprehensive character of the proposed methods, however, in this section we now turn our attention to the interactions of chaotic systems. These are an important group of systems characterized by randomlike but deterministic signals [60]. The intriguing dynamics of chaotic systems carry implications for many different fields, including, e.g., climate [61], ecology [62], and secure communications $[44,63]$. The application of the Bayesian inference and synchronization detection methods to chaotic systems follow the same procedure as that presented in Sec. II, based on the rationales included in Ref. [64]. In fact, the inference is even easier and more convenient to effect, because chaotic systems, with their strange attractors, typically visit more trajectories and thus facilitate inference within a larger volume of phase space. 
To detect PS and GS from interacting chaotic systems we considered the following Rössler system:

$$
\begin{aligned}
& \dot{x}_{1}=-1.8 x_{2}-x_{3} \\
& \dot{x}_{2}=1.8 x_{1}+0.3 x_{2}+\xi_{1} \\
& \dot{x}_{3}=0.5+x_{1} x_{3}-10 x_{3},
\end{aligned}
$$

which drives a Lorenz system

$$
\begin{aligned}
& \dot{y}_{1}=\sigma(t) y_{2}-10 y_{1} \\
& \dot{y}_{2}=\rho(t) y_{1}-y_{1} y_{3}-y_{2} \\
& \dot{y}_{3}=y_{1} y_{2}-2.66 y_{3}+\varepsilon(t) x_{2}+\xi_{2} .
\end{aligned}
$$

Three parameters of the Lorenz system are set to be time varying: sigma is continuously and periodically varied $\sigma(t)=$ $10+0.3 \sin (2 \pi 0.002 t)$, while the other two parameters are simultaneously and discreetly varied, i.e., the intrinsic parameter is $\rho(t)=\{0,28\}$ and the coupling parameters is $\varepsilon(t)=\{0,8\}$. The noise sources are again assumed to be white and Gaussian with strengths of $D_{1}=0.02$ and $D_{2}=0.5$.

We used the discreet parameters $\rho(t)$ and $\varepsilon(t)$ to control when the systems are synchronized and thus to introduce intermittent transitions. At first we set $\rho(t)=28$ and $\varepsilon(t)=0$. The two systems are then unsynchronized and each follows its own self-sustained dynamics. Next, when we set $\rho(t)=0$ and $\varepsilon(t)=8$, the Lorenz system becomes asymptotically stable and synchronized (in the generalized sense, as shown in Ref. [9]) to the Rössler dynamics. The latter statement can be proved by application of the second Lyapunov stability method to the dynamical error system $\mathbf{e}=\mathbf{y}-\mathbf{y}^{\prime}$, where $\mathbf{y}^{\prime}$ is an identical copy of $\mathbf{y}$ with different initial conditions [65]. By using the Lyapunov function $L=1 / 2\left(e_{1}^{2} / 10+e_{2}^{2}+e_{3}^{2}\right)$ one can derive that:

$$
\begin{aligned}
\dot{L} & =e_{1} / 10 \dot{e}_{1}+e_{2} \dot{e}_{2}+e_{3} \dot{e}_{3} \\
& =-\left(e_{1}-\frac{\sigma(t)}{20} e_{2}\right)^{2}-\left(1-\frac{\sigma(t)^{2}}{20^{2}}\right) e_{2}^{2}-2.66 e_{3}^{2} .
\end{aligned}
$$

If the variations of $\sigma(t)$ are confined to $\sigma(t) \in[-20,20]$, then $\dot{L}<0$, the driven system $\mathbf{y}$ is globally asymptotically stable and the two systems $\mathbf{x}$ and $\mathbf{y}$ are synchronized. We note that $\sigma(t)$ in our example was varied within the [9.7,10.3] interval and that the condition for asymptotic stability $\dot{L}<0$ was fulfilled. This provides an analytic proof for the existence of the synchronized state.

In order to verify whether the proposed methods can detect such a synchronized state, we applied them on signals generated from system Eqs. (10) and (11). By varying $\rho(t)$ and $\varepsilon(t)$ we introduced intermittent intervals of synchronization. Figure 6(a) shows a phase portrait of an unsynchronized interval, which transforms into well defined space structure once the systems undergo synchronization, as illustrated Fig. 6(b). In Fig. 6(c) we illustrate how well the inference can reconstruct the time-variations of the parameters $\varepsilon(t)$ and $\sigma(t)$. The evolution of the coupling parameter $\varepsilon(t)$ indicates when the synchronization intervals are introduced. The PS and GS of chaotic systems are usually equivalent [17], as in the example we present. In general, however, this may not be true so that exceptions can exist. For estimating the phases we used the analytic signals of $x_{2}$ and $y_{3}$ because
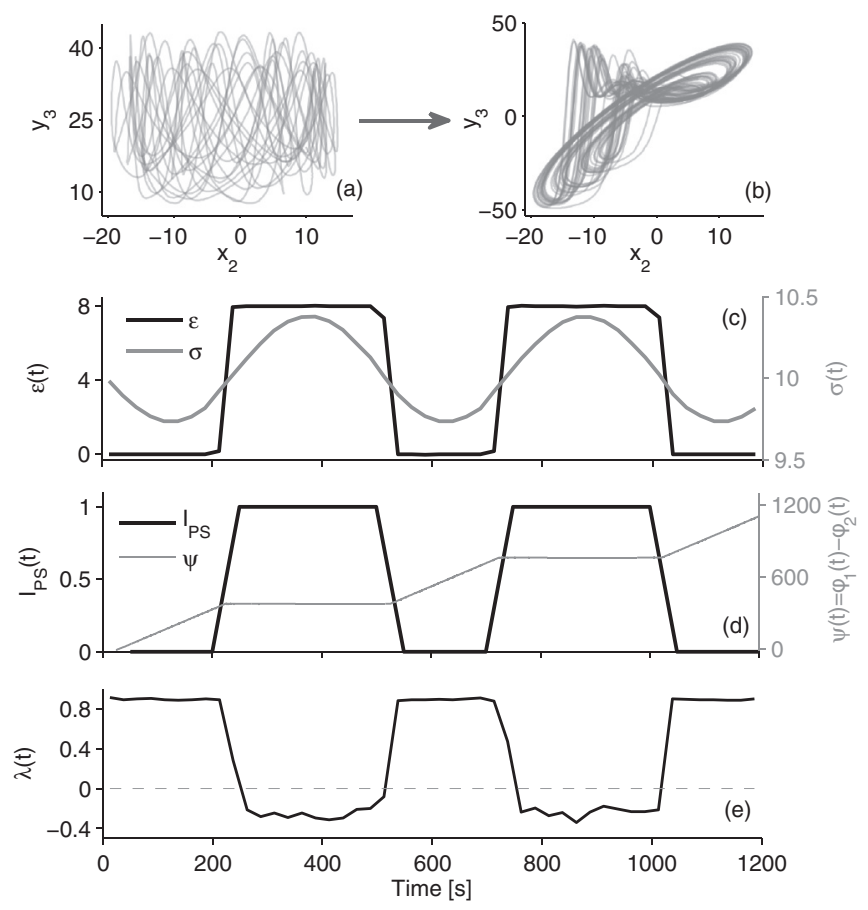

FIG. 6. Detection of PS and GS from interacting Rössler and Lorenz chaotic systems. Phase portrait of (a) nonsynchronized and (b) synchronized intervals. (c) Two inferred time-varying parameters $\sigma(t)$ and coupling $\epsilon(t)$. (d) Detected PS index $I_{\mathrm{PS}}$ and the corresponding instantaneous phase difference $\psi(t)$. (e) Detected LLE $\lambda$ as a measure of GS.

of their regular circular form. (Note that, in general, phase estimation from chaotic systems can be a nontrivial task and that special optimization techniques introduced in Ref. [41] may be needed). Application of the PS detection technique results in a synchronization index $I_{\mathrm{PS}}$ consistent with the phase difference and the deterministic transitions as shown in Fig. 6(d). By evaluating the LLE from the driven Lorenz system with the inferred parameters we are able to detect GS, as illustrated in Fig. 6(e). The transitions from positive to negative LLE are consistent with the deterministic parameters, the PS and the analytic condition Eq. (12).

\section{SUMMARY AND CONCLUSIONS}

In summary, we have demonstrated how PS and GS can be detected by the use of Bayesian dynamical inference. The evaluation was based on the core definitions of PS and GS, and it led to inference of parameter values in the models on which the procedure was being demonstrated. Thus, under the approximation that the noise is white and Gaussian, the method enabled the underlying stochastic differential models to be inferred from their time series. The resultant equations of motion describe the intrinsic mechanisms of interaction. For PS, the model consisted of coupled phase oscillators, using periodic Fourier series as base functions. For GS, a model consisting of polynomial base functions was used to represent the systems under investigation. We focused on systems that possess deterministic time-varying dynamics, recently formulated as chronotaxic [20], demonstrate that it can be 
inferred correctly, and show that the proposed methods can detect precisely the deterministic synchronization transitions characteristic of time-varying dynamics.

The two methods were illustrated and compared by means of analog electronic simulations, demonstrating that PS and GS can both be evaluated consistently despite the existence of time-varying perturbations. The experiment involved two unidirectionally coupled van der Pol oscillators, with timevarying characteristics, i.e., the frequency of the driving system was influenced by an external periodic signal. The electronic implementation and the experimental set-up can potentially be used for other studies involving intermittent synchronization of similar kinds. The dynamical noise in the signals originated from the real experimental conditions, i.e., from the circuit components. The proven effectiveness of the methods confirms that Bayesian inference of stochastic differential equations is both valid and desirable in the experimental context. The ability of the proposed methods to detect PS and GS within diverse classes of dynamical systems was further illustrated by their application to coupled chaotic systems. We studied a Rössler system that is intermittently driving a Lorenz chaotic system, with other time-varying parameters, and subject to noise. Correct detection was validated by comparison with the analytic synchronization condition.

In order to demonstrate the novelty and to differentiate our approach from other methods, we considered two important synchronization situations where the dynamical inference methods showed exceptional performance-in their abilities to detect noise-induced phase slips, and to distinguish coherence from coupling-induced synchronization. There were two main outcomes, both of which are crucially important for real-life, experimental situations. First, inference of the underlying stochastic dynamics allows intrinsic synchronization to be detected in cases where the presence of noise-induced phase slips might otherwise lead to the conclusion that the systems are unsynchronized. Second, inference of the deterministic dynamics facilitates discrimination between synchronization and phase coherence. In this way, possible spurious detection of synchronization from time series (or intervals of time series) that are not causally or functionally dependent can easily be identified. We compared the proposed methods with what are arguably the two most widely used methods for detection of PS [10] and GS [17]. The latter are easier to use and represent a whole class of similar methods based on statistical measures. Of course, our results in no way diminish the utility of these longer-established methods. Nonetheless, we have shown that, where dynamical inference methods are applicable, they can bring substantial advantages.

The methods described are particularly suitable for the analysis of data coming from thermodynamically open systems, subject to continuous external perturbations and noise, and with their own inherently time-varying dynamics. The cardiorespiratory interaction is an obvious and well-studied example ([4] and references therein). It is characterized by time variations in the frequencies of both the cardiac and respiratory oscillations, by their time-varying mutual coupling and by the presence of external noise. Hence, observed phase coherence may, or may not, be due to causal couplings in any given interval [66]. As such, systems abound in nature, we anticipate that the proposed methods will play an important role in terms of improved detection and deeper understanding of synchronization phenomena.

\section{ACKNOWLEDGMENTS}

We thank P. T. Clemson, T. Jüngling, and Y. F. Suprunenko for valuable discussions. This work was supported by the Engineering and Physical Sciences Research Council (U.K.) (Grant No. EP/100999X1).

\section{APPENDIX: INFERENCE OF THE MEASUREMENT MODEL}

The above analysis concentrated on the inference of how noise affects the dynamics because our interest focused on the detection of PS and GS in relation to dynamical noise-induced phase slips. In experiments, however, the observed time series $\mathcal{Y}_{n}$ can often also be affected by measurement or observational noise. These perturbations can reduce the overall precision of the dynamical inference. In such cases, one should infer both the dynamical Eq. (1), $\dot{\chi}_{i}=\mathbf{f}\left(\chi_{i}, \chi_{j} \mid \mathbf{c}\right)+\sqrt{\mathbf{D}} \xi_{i}$, and the measurement equation,

$$
\mathcal{Y}_{i}=\mathbf{g}\left(\chi_{i}, \chi_{j} \mid \mathbf{b}\right)+\sqrt{\mathbf{E}} \eta_{i},
$$

where $\mathbf{g}\left(\chi_{i}, \chi_{j} \mid \mathbf{b}\right)$ are measurement base functions parametrized by the $\mathbf{b}$ parameters, and the measurement noise $\eta_{i}$ is assumed to be white, Gaussian, and of intensity $\mathbf{E}$.

The aim now is to infer Eqs. (1) and (A1) under the common Bayesian framework. Using the same constraints (see Sec. II A and Ref. [33]) one can derive the log-likelihood function as

$$
\begin{aligned}
S= & S_{\text {dyn }}+S_{\text {meas }} \\
= & \frac{N}{2} \ln |\mathbf{D}|+\frac{h}{2} \sum_{n=0}^{N-1}\left\{\mathbf{c}_{k} \frac{\partial \mathbf{f}_{k}\left(\chi_{\cdot, n}\right)}{\partial \chi}\right. \\
& \left.+\left[\dot{\chi}_{n}-\mathbf{c}_{k} \mathbf{f}_{k}\left(\chi_{\cdot, n}^{*}\right)\right]^{T}\left(\mathbf{D}^{-1}\right)\left[\dot{\chi}_{n}-\mathbf{c}_{k} \mathbf{f}_{k}\left(\chi_{\cdot, n}^{*}\right)\right]\right\}+\frac{N}{2} \ln |\mathbf{E}| \\
& +\frac{1}{2} \sum_{n=0}^{N-1}\left[\mathcal{Y}_{n}-\mathbf{b}_{k} \mathbf{g}_{k}\left(\chi_{\cdot, n}\right)\right]^{T}\left(\mathbf{E}^{-1}\right)\left[\mathcal{Y}_{n}-\mathbf{b}_{k} \mathbf{g}_{k}\left(\chi_{\cdot, n}\right)\right] .
\end{aligned}
$$

Note that the first and the second term in the equation depict the inference of the dynamical model and are same as Eq. (3). The last two terms in the equation provide the (least-square-like) part of the likelihood function for inference of the measurement model. The priors are again given by the multivariate Gaussian distributions with the mean $\overline{\mathbf{c}}$ and covariance matrix $\Xi^{-1}$ prior for the dynamical, and by the mean $\overline{\mathbf{b}}$ and covariance matrix $\Theta_{\text {prior }}^{-1}$ for the measurement model. The parameters of the dynamical model are evaluated with the equations already given [see Eqs. (4)], while the measurement parameters are evaluated recursively with

$$
\begin{aligned}
\mathbf{E} & =\frac{1}{N}\left[\mathcal{Y}_{n}-\mathbf{b}_{k} \mathbf{g}_{k}\left(\chi_{\cdot, n}\right)\right]^{T}\left[\mathcal{Y}_{n}-\mathbf{b}_{k} \mathbf{g}_{k}\left(\chi_{\cdot, n}\right)\right], \\
\mathbf{b}_{k} & =\left(\Theta^{-1}\right)_{k w} \mathbf{u}_{w}, \\
\mathbf{u}_{w} & =\left(\Theta_{\text {prior }}\right)_{k w} \mathbf{b}_{w}+\mathbf{g}_{k}\left(\chi_{\cdot, n}\right)\left(\mathbf{E}^{-1}\right) \mathcal{Y}_{n}, \\
\Theta_{k w} & =\left(\Theta_{\text {prior }}\right)_{k w}+\mathbf{g}_{k}\left(\chi_{\cdot, n}\right)\left(\mathbf{E}^{-1}\right) \mathbf{g}_{w}\left(\chi_{\cdot, n}\right),
\end{aligned}
$$


where summation over $n=1, \ldots, N$ is assumed and the summation over repeated indices $k$ and $w$ is again implicit. Equations (4) and (A2) coupled with an optimization proce- dure in the paths' space represent the dynamical Bayesian framework for inference of nonlinear dynamical systems from measurements that are corrupted by noise.
[1] A. Pikovsky, M. Rosenblum, and J. Kurths, SynchronizationA Universal Concept in Nonlinear Sciences (Cambridge University Press, Cambridge, 2001).

[2] S. H. Strogatz, Sync: The Emerging Science of Spontaneous Order (Hyperion, New York, 2003).

[3] R. E. Mirollo and S. H. Strogatz, SIAM J. Appl. Math. 50, 1645 (1990).

[4] Y. Shiogai, A. Stefanovska, and P. V. E. McClintock, Phys. Rep. 488, 51 (2010).

[5] I. Z. Kiss, C. G. Rusin, H. Kori, and J. L. Hudson, Science 316, 1886 (2007).

[6] R. Brown and L. Kocarev, Chaos 10, 344 (2000).

[7] N. F. Rulkov, M. M. Sushchik, L. S. Tsimring, and H. D. I. Abarbanel, Phys. Rev. E 51, 980 (1995).

[8] M. G. Rosenblum, A. S. Pikovsky, and J. Kurths, Phys. Rev. Lett. 76, 1804 (1996).

[9] L. Kocarev and U. Parlitz, Phys. Rev. Lett. 76, 1816 (1996).

[10] P. Tass, M. G. Rosenblum, J. Weule, J. Kurths, A. Pikovsky, J. Volkmann, A. Schnitzler, and H.-J. Freund, Phys. Rev. Lett. 81, 3291 (1998).

[11] F. Mormann, K. Lehnertz, P. David, and C. E. Elger, Physica D 144, 358 (2000).

[12] B. Schelter, M. Winterhalder, R. Dahlhaus, J. Kurths, and J. Timmer, Phys. Rev. Lett. 96, 208103 (2006).

[13] B. Kralemann, A. Pikovsky, and M. Rosenblum, Phys. Rev. E 87, 052904 (2013).

[14] M. Paluš, V. Komárek, Z. Hrnčĩr̃, and K. Štěrbová, Phys. Rev. E 63, 046211 (2001).

[15] Z. Liu, J. Zhou, and T. Munakata, Europhys. Lett. 87, 50002 (2009).

[16] J. Schumacher, R. Haslinger, and G. Pipa, Phys. Rev. E 85, 056215 (2012).

[17] U. Parlitz, L. Junge, W. Lauterborn, and L. Kocarev, Phys. Rev. E 54, 2115 (1996)

[18] T. Stankovski, A. Duggento, P. V. E. McClintock, and A. Stefanovska, Phys. Rev. Lett. 109, 024101 (2012).

[19] A. Duggento, T. Stankovski, P. V. E. McClintock, and A. Stefanovska, Phys. Rev. E 86, 061126 (2012).

[20] Y. F. Suprunenko, P. T. Clemson, and A. Stefanovska, Phys. Rev. Lett. 111, 024101 (2013).

[21] M. D. Chekroun, E. Simonnet, and M. Ghil, Physica D 240, 1685 (2011).

[22] P. M. de Oliveira, Theor. Biosci. 120, 1 (2001).

[23] K. J. Friston, NeuroImage 16, 513 (2002).

[24] V. N. Smelyanskiy, D. G. Luchinsky, A. Stefanovska, and P. V. E. McClintock, Phys. Rev. Lett. 94, 098101 (2005).

[25] U. von Toussaint, Rev. Mod. Phys. 83, 943 (2011).

[26] K. Friston, R. Moran, and A. K. Seth, Curr. Opin. Neurobiol. 23, 172 (2013).

[27] D. Marinazzo, M. Pellicoro, and S. Stramaglia, Phys. Rev. Lett. 100, 144103 (2008).

[28] L. Barnett, A. B. Barrett, and A. K. Seth, Phys. Rev. Lett. 103, 238701 (2009).
[29] M. Staniek and K. Lehnertz, Phys. Rev. Lett. 100, 158101 (2008).

[30] E. Bullmore and O. Sporns, Nat. Rev. Neurosci. 10, 186 (2009).

[31] L. Faes, G. Nollo, and A. Porta, Phys. Rev. E 83, 051112 (2011).

[32] K. J. Friston, Brain Connect. 1, 13 (2011).

[33] D. G. Luchinsky, V. N. Smelyanskiy, A. Duggento, and P. V. E. McClintock, Phys. Rev. E 77, 061105 (2008).

[34] B. Kralemann, L. Cimponeriu, M. Rosenblum, A. Pikovsky, and R. Mrowka, Phys. Rev. E 77, 066205 (2008).

[35] H. U. Voss, J. Timmer, and J. Kurths, Int. J. Bifurcat. Chaos 14, 1905 (2004).

[36] J. Daunizeau, K. J. Friston, and S. J. Kiebel, Physica D 238, 2089 (2009).

[37] D. A. Smirnov and B. P. Bezruchko, Phys. Rev. E 79, 046204 (2009).

[38] T. Stankovski, A. Duggento, P. V. E. McClintock, and A. Stefanovska, arXiv:1305.0041.

[39] D. Gabor, J. IEEE 93, 429 (1946).

[40] I. Daubechies, J. Lu, and H. Wu, Appl. Comput. Harmon. Anal. 30, 243 (2011).

[41] J. T. C. Schwabedal and A. Pikovsky, Phys. Rev. Lett. 110, 204102 (2013).

[42] Y. Kuramoto, Chemical Oscillations, Waves, and Turbulence (Springer-Verlag, Berlin, 1984).

[43] H. Attias, in Advances in Neural Information Processing Systems, edited by S. Solla, T. Leen, and K. Muller (2000), Vol. 12 of Advances in Neural Information Processing Systems, pp. 209, 13th Annual Conference on Neural Information Processing Systems (NIPS), CO, Nov 29-Dec 04, 1999.

[44] T. Stankovski, P. V. E. McClintock, and A. Stefanovska, Phys. Rev. X 4, 011026 (2014).

[45] J. P. Eckmann and D. Ruelle, Rev. Mod. Phys. 57, 617 (1985).

[46] A. Wolf, J. B. Swift, H. L. Swinney, and J. A. Vastano, Physica D 16, 285 (1985).

[47] D. G. Luchinsky, P. V. E. McClintock, and M. I. Dykman, Rep. Prog. Phys. 61, 889 (1998).

[48] D. G. Luchinsky and P. V. E. McClintock, Nature 389, 463 (1997).

[49] A. G. Balanov, N. B. Janson, D. E. Postnov, and P. V. E. McClintock, Phys. Rev. E 65, 041105 (2002).

[50] A. A. Temirbayev, Z. Z. Zhanabaev, S. B. Tarasov, V. I. Ponomarenko, and M. Rosenblum, Phys. Rev. E 85, 015204 (2012).

[51] T. L. Carroll and L. M. Pecora, IEEE Trans. Circuits Syst. II 40, 646 (1993).

[52] T. Stankovski, Tackling the Inverse Problem for NonAutonomous Systems: Application to the Life Sciences (Springer, Berlin, 2013).

[53] G. Kaiser, A Friendly Guide to Wavelets (Birkhäuser, Boston, 1994).

[54] A. Stefanovska, M. Bračič, and H. D. Kvernmo, IEEE Trans. Bio. Med. Eng. 46, 1230 (1999). 
[55] See Supplemental Material at http://link.aps.org/supplemental/ 10.1103/PhysRevE.89.062909 for Video 1 showing in real time the Lissajou curve on the oscilloscope with continuous timevariation and synchronization transitions.

[56] A. Aragoneses, N. Rubido, J. Tiana-Alsina, M. C. Torrent, and C. Masoller, Sci. Rep. 3, 1778 (2013).

[57] J. N. Teramae and D. Tanaka, Phys. Rev. Lett. 93, 204103 (2004).

[58] M. Vejmelka and M. Paluš, Phys. Rev. E 77, 026214 (2008).

[59] J. Theiler, S. Eubank, A. Longtin, B. Galdrikian, and J. Farmer, Physica D 58, 77 (1992).
[60] S. H. Strogatz, Nature 410, 268 (2001).

[61] E. N. Lorenz, J. Atmos. Sci. 20, 130 (1963).

[62] B. Blasius, A. Huppert, and L. Stone, Nature 399, 354 (1999).

[63] K. M. Cuomo and A. V. Oppenheim, Phys. Rev. Lett. 71, 65 (1993).

[64] V. N. Smelyanskiy, D. G. Luchinsky, D. A. Timucin, and A. Bandrivskyy, Phys. Rev. E 72, 026202 (2005).

[65] R. He and P. G. Vaidya, Phys. Rev. A 46, 7387 (1992).

[66] D. Iatsenko, A. Bernjak, T. Stankovski, Y. Shiogai, P. J. Owen-Lynch, P. B. M. Clarkson, P. V. E. McClintock, and A. Stefanovska, Phil. Trans. R. Soc. Lond. A 371, 20110622 (2013). 